\title{
Synthesis and Characterization of 2-Butoxyethylxanthate Complexes with Iron(II), Cobalt(II), Nickel(II), Copper(II) and Zinc(II) and their Adducts with Nitrogen Base Ligands
}

\author{
Saad E. Al-Mukhtar Ibraheem A. Al-Qasser \\ Nada F. Hana \\ Department of Chemistry \\ College of Science \\ University of Mosul
}

(Received 29/4/2013; Accepted 9/9/2013)

\begin{abstract}
New complexes and adducts of xanthate of the general formula [M(2-BuoEtxant $\left.)_{2}\right]$ and $\left[\mathrm{M}(2-B u o E t x a n t)_{2} \cdot \mathrm{nL}\right]$ Where $\mathrm{M}=\mathrm{Fe}(\mathrm{II}), \mathrm{Co}(\mathrm{II}), \mathrm{Ni}(\mathrm{II}), \mathrm{Cu}(\mathrm{II})$ and $\mathrm{Zn}(\mathrm{II})$, and (2-BuoEtxant) $=2$-Butoxyethylxanthate, and $\mathrm{n}=2$ when $\mathrm{L}=$ pyridine; Isoquinoline, $\gamma$-picoline, 3,5-lutidine, $\mathrm{n}=1$ when $\mathrm{L}=$ ethylenediamine, $(1,10)$-phenanthroline, have been prepared and characterized by metal analyses, infrared, conductance measurements, electronic absorption spectra and susceptibility measurements. Magnetic moment and electronic spectra, indicate that the complexes of type $\left[\mathrm{M}(2-\text { BuoEtxant })_{2}\right]$ are of tetrahedral geometry while the complexes of type $\left[\mathrm{M}(2-\mathrm{BuoEtxant})_{2 .} \mathrm{nL}\right]$ have an octahedral geometry.

Keywords: Xanthate, Iron(II), Cobalt(II), Nickel(II), Copper(II), Zinc(II), Complexes.

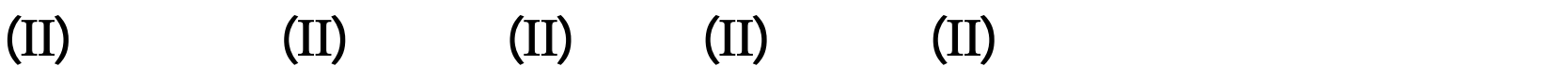 مع 2 -بيوتوكس الثل زلثيت ومركبلت الاضفةمع عددمن الفواعد النتروجينية \\ الملغص}

حضرت معقدات جنية ذات ال صيغة [M(2-BuoEtxant) و[M(2-BuoEtxant)2.nL] حي ث ان 2-BuoEtxant) و 2 [ Zn(II),Cu(II),Ni(II),Co(II),Fe(II) =M]

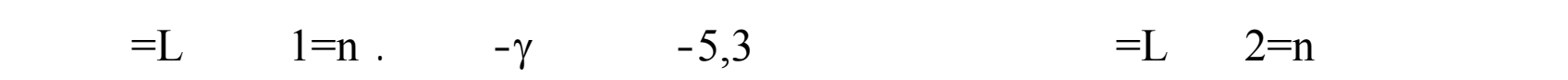

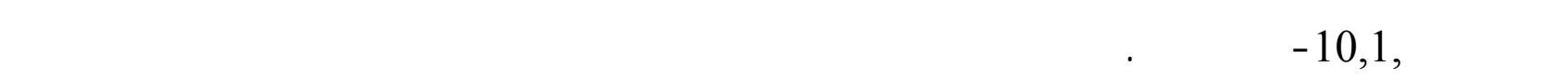

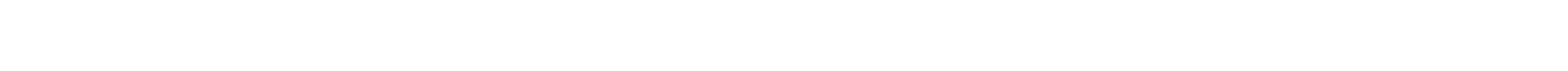
والطف الالكتروني على التخاذشكل رباعي للنطوح للمعدات ذات ال ـصيغة [M(2-BuoEtxant) التخذت المعقداتسدلسية التنلسق ذات الصيغة [M(2-BuoEtxant)2.nL]شكل ثماني المطوح.
\end{abstract}




\section{INTRODUCTION}

Transition matel complexes in which a 1,1-dithio ligand form a four membered ring with the metal ion have been extensively investigated and thoroughly reviewed (Coucouvanis, 1979; Bond and Martin,1984; Cox, et al.,1997; Therm,1994). The four membered ring metal complexes expand their coordination number by interaction with a lewis base. This may take place by adduct formation with other ligands of comparable ligating ability. The resulting complex is significantly different from those of the complex not having the expanded coordination number (Magnus et al., 1994; Coucouvanis, 1979).

2-Boutoxyethylxanthates, $\mathrm{S}_{2}^{-} \mathrm{COR}$, comprise an important class of 1,1-dithiolate ligands with many applications ranging from flotation agent to radical polymerization (Coucouvanis,1970; Coucouvanis, 1979; Coote and Radom, 2004; Wan, et al., 2005; Tiekink and Haiduc, 2005).

Thus, we have described the preparation of some Fe(II), Co(II), Ni(II), $\mathrm{Cu}(\mathrm{II}), \mathrm{Zn}$ (II) complexes containing 2-butoxyethyxanthate ligand and their adducts with nitrogen bases . The nitrogen bases stabilize the binary complexes. The isolated products are stable in air but their stability decreased markedly when dissolved in organic solvents.

\section{Materials and Methods}

\section{EXPERIMENTAL}

All reagents and solvents were of analytical grade and used as supplied from Fluka or BDH chemical companies. Infrared spectra were recorded on a Brucker Tensor 27co. FTIR spectrophotometer in the $400-4000 \mathrm{~cm}^{-1}$ range using $\mathrm{KBr}$ discs. Conductivity measurements were carried out on a $10^{-3} \mathrm{M}$ solution of the complexes in DMF using conductivity meter PCM3 Jenway at ambient temperature. The electronic spectra were recorded on a Shimadzu UV-visible spectrophotometer UV-160 for $10^{-3} \mathrm{M}$ solution of complexes in DMF as solvent at $25^{\circ} \mathrm{C}$ using $1 \mathrm{~cm}$ quartz cells. Metals content was determined using AA670 atomic absorption. Melting points were recorded on an Electrothermal 9300 apparatus and were uncorrected. The magnetic measurement was carried out at $25^{\circ} \mathrm{C}$ on the solids by Faraday's method using Brucker BM6 instrument.

\section{Synthesis of potassium 2-Butoxyethylxanthate}

2-Butoxyethanol $(11.80 \mathrm{~g}, 0.10 \mathrm{~mol})$ was added to aqueous solution $(5.61 \mathrm{~g}, 0.10 \mathrm{~mol})$ of potassium hydroxide with stirring. The mixture was cooled in an ice bath, to this mixture $\left(7.60 \mathrm{~cm}^{3}, 0.10 \mathrm{~mol}\right)$ of carbon disulfide was added dropwise with continuous stirring for 30 min. in ice bath, the yellow precipitate formed was filtered off, washed with diethylether and dried under vacuum.

\section{I.A. Synthesis of complexes [M (2-BuoEtxant $\left.)_{2}\right]$ \\ $\mathrm{M}=\mathrm{Fe}(\mathrm{II}), \mathrm{Co}(\mathrm{II}), \mathrm{Ni}(\mathrm{II}), \mathrm{Cu}(\mathrm{II}), \mathrm{Zn}(\mathrm{II})$}

An ethanolic solution of $\mathrm{FeCl}_{2} \cdot 4 \mathrm{H}_{2} \mathrm{O}(1.98 \mathrm{~g}, 0.01 \mathrm{~mol})$ or $\mathrm{CoCl}_{2} \cdot 6 \mathrm{H}_{2} \mathrm{O}(2.37 \mathrm{~g}, 0.01 \mathrm{~mol})$ or $\mathrm{NiCl}_{2} .6 \mathrm{H}_{2} \mathrm{O}(2.37 \mathrm{~g}, 0.01 \mathrm{~mol})$ or $\mathrm{CuCl}_{2} .2 \mathrm{H}_{2} \mathrm{O}(1.70 \mathrm{~g}, 0.01 \mathrm{~mol})$ or $\mathrm{ZnCl}_{2}(1.36 \mathrm{~g}, 0.01 \mathrm{~mol})$ was added dropwise to an ethanolic solution of potassium 2-butoxyethylxanthate $(2.36 \mathrm{~g}$, $0.02 \mathrm{~mol}$ ) with stirring for $30 \mathrm{~min}$ until complete precipitation. The precipitate was filtered off, washed with ethanol, then with diethylether and dried under vacuum. 


\section{I.B. Synthesis of complexes [M (2-BuoEtxant $\left.)_{2}(\mathrm{~L})_{2}\right]$ \\ $\mathrm{L}=$ pyridine, isoquinoline, $\gamma$-picoline, 3,5 -lutidine}

Prepared similarly as in (I.A.). The precipitate formed was treated with $(0.02 \mathrm{~mol})$ of (pyridine, isoquinoline, $\gamma$-picoline, 3,5-lutidine) dropwise with continuous stirring for 30 min., the precipitate formed was filtered off and washed with ethanol then dried under vacuum.

\section{I.C. Synthesis of complexes [M (2-BuoEtxant $\left.)_{2}(\mathrm{~L})\right]$ $L=1,10$-phenanthroline,ethylenediamine}

Prepared similarly as in (I.A.) and the precipitate formed was treated with $(0.01 \mathrm{~mol})$ of (1,10-phenanthroline, ethylenediamine) dropwise with continuous stirring for $30 \mathrm{~min}$., the precipitate formed was, filtered, washed with ethanol, then dried under vacuum.

\section{RESULTS AND DISCUSSTION}

The new ligand was prepared by the reaction of 2-butoxyethanol with potassium hydroxide and carbon disulphide: the complexes were prepared through direct reaction of the metal chlorides, $\mathrm{FeCl}_{2} \cdot 4 \mathrm{H}_{2} \mathrm{O}, \mathrm{CoCl}_{2} \cdot 6 \mathrm{H}_{2} \mathrm{O}, \mathrm{NiCl}_{2} \cdot 6 \mathrm{H}_{2} \mathrm{O}, \mathrm{CuCl}_{2} .2 \mathrm{H}_{2} \mathrm{O}$ or unhydrous $\mathrm{ZnCl}_{2}$ with the above ligand in (1:2) molar ratio. The values of conductivity measurement in dimethylformamide solution of the complexes are in the range (2.30-38.30) $\Omega^{-1} . \mathrm{cm}^{2} . \mathrm{mol}^{-1}$, which are typical values for non electrolyte type (Geary,1971).

\section{Magnetic susceptibility measurements}

The magnetic moments of the complexes Table (1) were measured at $\left(25^{\circ} \mathrm{C}\right)$. The magnetic moments for $\mathrm{Fe}(\mathrm{II}), \mathrm{Co}(\mathrm{II}), \mathrm{Ni}(\mathrm{II})$ and $\mathrm{Cu}(\mathrm{II})$ complexes $(1,8,15$ and 22) (2.004.98 B.M) suggested a tetrahedral geometry (Nicholls, 1973). The magnetic moments values of the other complexes $(2-7,9-14,16-21,23-28)$ were in the range (1.58-5.36 B.M.) in an octahedral geometry. The high values of magnetic moments of some octahedral $\mathrm{Co}$ (II) complexes from the spin only value are due to the orbital contribution. (Nicholls, 1973).

\section{Electronic spectral studies}

The UV-Visible spectra of the ligand and its complexes of $10^{-3} \mathrm{M}$ solution in DMF were recorded; the results were listed in Table (2). the UV-Visible spectra of the $\mathrm{Fe}(\mathrm{II})$ complex (1) gives absorption band at $\left(9842 \mathrm{~cm}^{-1}\right)$, which corresponds to $\left({ }^{5} \mathrm{E} \rightarrow{ }^{5} \mathrm{~T}_{2}\right)$ transition in a tetrahedral geometry (Nicholls, 1973). Complexes (2-7) show absorption band at the range $\left(10217-11037 . \mathrm{cm}^{-1}\right)$, which were assigned to $\left({ }^{5} \mathrm{~T}_{2} \mathrm{~g} \rightarrow{ }^{5} \mathrm{Eg}\right)$ transition these observations indicating an octahedral geometry around $\mathrm{Fe}(\mathrm{II})$ ions (Coucouvanis and Fackler, 1967).

The Co(II) complex (8) exhibited an absorption band at $\left(15544 \mathrm{~cm}^{-1}\right)$ region, which was assigned to $\left({ }^{4} \mathrm{~A}_{2}(\mathrm{~F}) \rightarrow{ }^{4} \mathrm{~T}_{1}(\mathrm{P})\right)$ transition (Siddiqi and Nishat, 2000), in a tetrahedral configuration of this complex and the absence of $\left({ }^{4} \mathrm{~A}_{2}(\mathrm{~F}) \rightarrow{ }^{4} \mathrm{~T}_{2}(\mathrm{~F})\right) \operatorname{and}\left({ }^{4} \mathrm{~A}_{2}(\mathrm{~F}) \rightarrow{ }^{4} \mathrm{~T}_{1}(\mathrm{~F})\right)$, are due to the sensitivity of the instrument used. Complexes (9-14) show three absorption bands at the range $\left(9823-11086 \mathrm{~cm}^{-1}\right),\left(14124-16339 \mathrm{~cm}^{-1}\right)$ and $\left(20024-21777 \mathrm{~cm}^{-1}\right)$, which were assigned to $\left({ }^{4} \mathrm{~T}_{1} \mathrm{~g}(\mathrm{~F}) \rightarrow{ }^{4} \mathrm{~T}_{2} \mathrm{~g}(\mathrm{~F})\right),\left({ }^{4} \mathrm{~T}_{1} \mathrm{~g}(\mathrm{~F}) \rightarrow{ }^{4} \mathrm{~A}_{2} \mathrm{~g}(\mathrm{~F})\right)$ and $\left({ }^{4} \mathrm{~T}_{1} \mathrm{~g}(\mathrm{~F}) \rightarrow{ }^{4} \mathrm{~T}_{1} \mathrm{~g}(\mathrm{P})\right)$ transition respectively in an octahedral configuration (Martel, 1971; Onwudiwe and Ajibade, 2010).

The Ni(II) complex (15) show two absorption bands at $\left(9433 \mathrm{~cm}^{-1}\right)$ and $\left(15290 \mathrm{~cm}^{-1}\right)$ which were assigned to $\left({ }^{3} \mathrm{~T}_{1}(\mathrm{~F}) \rightarrow{ }^{3} \mathrm{~A}_{2}(\mathrm{~F})\right)$ and $\left({ }^{3} \mathrm{~T}_{1}(\mathrm{~F}) \rightarrow{ }^{3} \mathrm{~T}_{1}(\mathrm{P})\right)$ transitions respectively in a 
tetrahedral geometry ( Nicholls, 1973), the complexes (16-21) show three absorption bands in the range $\left(9363-10460 \mathrm{~cm}^{-1}\right),\left(15197-16447 \mathrm{~cm}^{-1}\right)$ and $\left(21739-27472 \mathrm{~cm}^{-1}\right)$, which were assigned $\left({ }^{3} \mathrm{~A}_{2} \mathrm{~g}(\mathrm{~F}) \rightarrow{ }^{3} \mathrm{~T}_{2} \mathrm{~g}(\mathrm{~F})\right),\left({ }^{3} \mathrm{~A}_{2} \mathrm{~g}(\mathrm{~F}) \rightarrow{ }^{3} \mathrm{~T}_{1} \mathrm{~g}(\mathrm{~F})\right)$ and $\left({ }^{3} \mathrm{~A}_{2} \mathrm{~g}(\mathrm{~F}) \rightarrow{ }^{3} \mathrm{~T}_{1} \mathrm{~g}(\mathrm{P})\right)$ respectively, which are characteristic for an octahedral geometry Ni(II) complexes (Singh et al., 1989).

The $\mathrm{Cu}(\mathrm{II})$ complex (22) gives an absorption band at $\left(9823 \mathrm{~cm}^{-1}\right)$, corresponds to $\left({ }^{2} \mathrm{~T}_{2} \rightarrow{ }^{2} \mathrm{E}\right)$ transition in a tetrahedral geometry (Cookson et al., 2010), while the complexes (23-28) show a broad absorption band in the region (13054-13886 $\mathrm{cm}^{-1}$ ), which was assigned to $\left({ }^{2} \mathrm{Eg} \rightarrow{ }^{2} \mathrm{~T}_{2} \mathrm{~g}\right)$ transition which may be formed from the combination of three transitions $\left({ }^{2} \mathrm{~B}_{1} \mathrm{~g} \rightarrow{ }^{2} \mathrm{~A}_{1} \mathrm{~g}\right),\left({ }^{2} \mathrm{~B}_{1} \mathrm{~g} \rightarrow{ }^{2} \mathrm{~B}_{2} \mathrm{~g}\right)$ and $\left({ }^{2} \mathrm{~B}_{1} \mathrm{~g} \rightarrow{ }^{2} \mathrm{Eg}\right)$ in disturted octahedral $\mathrm{Cu}(\mathrm{II})$ complexes. (Lever et al.,1984 ; Sarwar et al., 2007).

For all compounds the other bands are refering to charge transfer and ligand transitiens.

\section{Infra-red spectral studies}

The important IR bands of the ligand (2-BuoEtXant)K and their complexes are listed in Table (2). The two bonds $v(\mathrm{C}-\mathrm{O})$ and $v(\mathrm{C}-\mathrm{S})$ of the ligand were observed at $1028 \mathrm{~cm}^{-1}$ and $1099 \mathrm{~cm}^{-1}$, in all complexes these bond shift to appear at the region $(1130-1269) \mathrm{cm}^{-1}$ and (1028-1072) $\mathrm{cm}^{-1}$ respectively. The presence of only one band in the later region reports the bidentate coordination of the dithio ligand (Serrano, 2003).

IR spectra showed a new band at (415-440) $\mathrm{cm}^{-1}$ which may be an is the evidence for the coordination of metal to sulfur $v(\mathrm{M}-\mathrm{S})$. This behavior may be attributed to the electronreleasing of the alcohol, which forces high electron density towards the sulfur atoms (Rathore et al., 2007).

Table 1: Analytical and some physical properties of the prepared complexes

\begin{tabular}{|c|c|c|c|c|c|c|c|}
\hline No. & Compound & Color & m.p $\left({ }^{\circ} \mathbf{c}\right)$ & $\begin{array}{l}\text { Molar conductivity } \\
\Omega^{-1} \cdot \mathrm{cm}^{2} \cdot \mathrm{mol}^{-1}\end{array}$ & $\% \mathbf{M}$ & $\%$ yield & $\begin{array}{c}\mu_{e f f} \\
\text { B.M }\end{array}$ \\
\hline & (2-BuoEtXant)K & yellow & & ---- & ---- & $\begin{array}{cl}--- \\
\end{array}$ & --- \\
\hline 1 & {$\left[\mathrm{Fe}(2-\mathrm{BuoEtXant})_{2}\right]$} & Orange & 240 & 24.5 & $\begin{array}{c}12.99 \\
(12.63) \\
\end{array}$ & 18 & 4.98 \\
\hline 2 & {$\left[\mathrm{Fe}(2-\mathrm{BuoEtXant})_{2} \cdot(\mathrm{py})_{2}\right]$} & Orange & 200 & 38.3 & $\begin{array}{c}9.86 \\
(9.31)\end{array}$ & 56 & 5.30 \\
\hline 3 & {$\left[\mathrm{Fe}(2-\mathrm{BuoEtXant})_{2} \cdot(4-\mathrm{pic})_{2}\right]$} & Light brown & 185 & 25.3 & $\begin{array}{c}9.55 \\
(8.89)\end{array}$ & 70 & 5.03 \\
\hline 4 & $\left.\left[\mathrm{Fe}(2-\mathrm{BuoEtXant})_{2} \text {.(iso-Q }\right)_{2}\right]$ & Orange & $100^{*}$ & 35.7 & $\begin{array}{c}8.45 \\
(7.98)\end{array}$ & 87 & 5.36 \\
\hline 5 & {$\left[\mathrm{Fe}(2-\mathrm{BuoEtXant})_{2} \cdot(3,5-\mathrm{lut})_{2}\right]$} & Orange & $260^{*}$ & 31.3 & $\begin{array}{c}9.01 \\
(8.51)\end{array}$ & 57 & 5.19 \\
\hline 6 & {$\left[\mathrm{Fe}(2-\mathrm{BuoEtXant})_{2} \cdot(1,10\right.$-phen $\left.)\right]$} & Brown & 180 & 18.5 & $\begin{array}{c}9.63 \\
(8.98)\end{array}$ & 65 & 4.95 \\
\hline 7 & {$\left[\mathrm{Fe}(2-\mathrm{BuoEtXant})_{2} \cdot(\mathrm{en})\right]$} & Light purple & 160 & 35.3 & $\begin{array}{l}11.89 \\
(11.13)\end{array}$ & 97 & 4.83 \\
\hline 8 & {$\left[\mathrm{Co}(2 \text {-BuoEtXant })_{2}\right]$} & Dark green & 270 & 8.5 & $\begin{array}{c}11.92 \\
(11.31) \\
\end{array}$ & 71 & 3.99 \\
\hline 9 & {$\left[\mathrm{Co}(2-\mathrm{BuoEtXant})_{2} \cdot(\mathrm{py})_{2}\right]$} & Dark brown & 240 & 2.3 & $\begin{array}{c}9.05 \\
(8.58)\end{array}$ & 82 & 4.67 \\
\hline 10 & {$\left[\mathrm{Co}(2-\mathrm{BuoEtXant})_{2} \cdot(4-\text { pic })_{2}\right]$} & Dark brown & 190 & 4.3 & $\begin{array}{c}8.79 \\
(8.21)\end{array}$ & 78 & 4.50 \\
\hline
\end{tabular}


Synthesis and Characterization.......

\begin{tabular}{|c|c|c|c|c|c|c|c|}
\hline 11 & {$\left[\mathrm{Co}(2-\mathrm{BuoEtXant})_{2} \cdot(\text { iso-Q })_{2}\right]$} & Dark brown & $200^{*}$ & 24.6 & $\begin{array}{c}7.94 \\
(7.43) \\
\end{array}$ & 76 & 4.36 \\
\hline 12 & {$\left[\mathrm{Co}(2-\mathrm{BuoEtXant})_{2} \cdot(3,5 \text {-lut })_{2}\right]$} & Dark brown & 270 & 8.6 & $\begin{array}{c}8.56 \\
(7.89)\end{array}$ & 57 & 4.73 \\
\hline 13 & {$\left[\mathrm{Co}(2-\mathrm{BuoEtXant})_{2} \cdot(1,10-\right.$ phen $\left.)\right]$} & Black & 280 & 3.6 & $\begin{array}{c}8.88 \\
(8.29)\end{array}$ & 83 & 4.77 \\
\hline 14 & {$\left[\mathrm{Co}(2-\mathrm{BuoEtXant})_{2} \cdot(\mathrm{en})\right]$} & Dark brown & $220^{*}$ & 6.5 & $\begin{array}{c}10.67 \\
(10.08)\end{array}$ & 59 & 4.15 \\
\hline 15 & {$\left[\mathrm{Ni}(2-\mathrm{BuoEtXant})_{2}\right]$} & Dark green & $200^{*}$ & 12.9 & $\begin{array}{c}13.86 \\
(13.25)\end{array}$ & 37 & 3.35 \\
\hline 16 & {$\left[\mathrm{Ni}(2-\mathrm{BuoEtXant})_{2} \cdot(\mathrm{py})_{2}\right]$} & Orange & $65^{*}$ & 13.7 & $\begin{array}{c}10.33 \\
(9.77)\end{array}$ & 88 & 2.88 \\
\hline 17 & {$\left[\mathrm{Ni}(2-\mathrm{BuoEtXant})_{2} \cdot(\text { 4-pic })_{2}\right]$} & Dark green & 140 & 16.6 & $\begin{array}{c}10.23 \\
(9.34)\end{array}$ & 86 & 3.13 \\
\hline 18 & {$\left[\mathrm{Ni}(2-\mathrm{BuoEtXant})_{2} \cdot(\text { iso-Q })_{2}\right]$} & Dark green & $125^{*}$ & 18.6 & $\begin{array}{c}9.12 \\
(8.38)\end{array}$ & 77 & 2.94 \\
\hline 19 & {$\left[\mathrm{Ni}(2-\mathrm{BuoEtXant})_{2} \cdot(3,5-\mathrm{lut})_{2}\right]$} & Dark green & $110^{*}$ & 25.6 & $\begin{array}{c}9.67 \\
(8.94)\end{array}$ & 90 & 2.82 \\
\hline 20 & {$\left[\mathrm{Ni}(2-\mathrm{BuoEtXant})_{2} \cdot(1,10\right.$-phen $\left.)\right]$} & Light purple & $125^{*}$ & 7.1 & $\begin{array}{c}9.94 \\
(9.43)\end{array}$ & 46 & 2.97 \\
\hline 21 & {$\left[\mathrm{Ni}(2-\mathrm{BuoEtXant})_{2} \cdot(\mathrm{en})\right]$} & Dark green & 220 & 6.3 & $\begin{array}{c}11.97 \\
(11.67)\end{array}$ & 69 & 3.00 \\
\hline 22 & {$\left[\mathrm{Cu}(2-\mathrm{BuoEtXant})_{2}\right]$} & Dark brown & $155^{*}$ & 3.3 & $\begin{array}{c}12.12 \\
(11.86)\end{array}$ & 81 & 2.00 \\
\hline 23 & 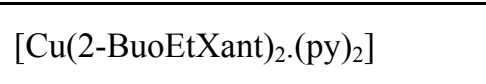 & Light yellow & 140 & 11.4 & $\begin{array}{c}9.45 \\
(8.99)\end{array}$ & 70 & 1.84 \\
\hline 24 & {$\left[\mathrm{Cu}(2-\mathrm{BuoEtXant})_{2} \cdot(4-\mathrm{pic})_{2}\right]$} & Green yellow & $170^{*}$ & 5.4 & $\begin{array}{c}9.01 \\
(8.63)\end{array}$ & 37 & 2.52 \\
\hline 25 & {$\left[\mathrm{Cu}(2-\mathrm{BuoEtXant})_{2} \cdot(\text { iso-Q })_{2}\right]$} & Brown & $140^{*}$ & 27.1 & $\begin{array}{c}8.44 \\
(7.81)\end{array}$ & 26 & 1.65 \\
\hline 26 & {$\left[\mathrm{Cu}(2 \text {-BuoEtXant })_{2} \cdot(3,5 \text {-lut })_{2}\right]$} & Brown & 120 & 31.8 & $\begin{array}{c}8.74 \\
(8.29)\end{array}$ & 68 & 2.10 \\
\hline 27 & {$\left[\mathrm{Cu}(2-\mathrm{BuoEtXant})_{2} \cdot(1,10\right.$-phen $\left.)\right]$} & Light brown & $172^{*}$ & 7.6 & $\begin{array}{c}9.61 \\
(8.71)\end{array}$ & 77 & 1.58 \\
\hline 28 & {$\left[\mathrm{Cu}(2-\mathrm{BuoEtXant})_{2} \cdot(\mathrm{en})\right]$} & Yellow & 110 & 12.5 & $\begin{array}{c}10.89 \\
(10.58)\end{array}$ & 80 & 2.22 \\
\hline 29 & {$\left[\mathrm{Zn}(2-\text { BuoEtXant })_{2}\right]$} & White & $120^{* *}$ & 8.2 & $\begin{array}{c}13.65 \\
(13.20)\end{array}$ & 63 & Dia \\
\hline 30 & {$\left[\mathrm{Zn}(2-\mathrm{BuoEtXant})_{2} \cdot(\mathrm{py})_{2}\right]$} & White & $300^{* *}$ & 34.7 & $\begin{array}{l}10.49 \\
(9.74)\end{array}$ & 76 & Dia \\
\hline 31 & {$\left[\mathrm{Zn}(2-\mathrm{BuoEtXant})_{2} \cdot(\text { 4-pic })_{2}\right]$} & Light yellow & $300^{* *}$ & 10.7 & $\begin{array}{c}9.74 \\
(9.31)\end{array}$ & 26 & Dia \\
\hline 32 & {$\left[\mathrm{Zn}(2-\mathrm{BuoEtXant})_{2} \cdot(\text { iso-Q })_{2}\right]$} & White & $300^{*}$ & 33.7 & $\begin{array}{c}8.90 \\
(8.35)\end{array}$ & 86 & Dia \\
\hline 33 & {$\left[\mathrm{Zn}(2 \text {-BuoEtXant })_{2} \cdot(3,5 \text {-lut })_{2}\right]$} & Light yellow & $75^{*}$ & 2.8 & $\begin{array}{c}9.47 \\
(8.90)\end{array}$ & 38 & Dia \\
\hline 34 & [Zn(2-BuoEtXant) $)_{2} \cdot(1,10$-phen $\left.)\right]$ & Yellow & $160^{*}$ & 31.1 & $\begin{array}{c}9.81 \\
(9.39)\end{array}$ & 46 & Dia \\
\hline 35 & {$\left[\mathrm{Zn}(2-\mathrm{BuoEtXant})_{2} \cdot(\mathrm{en})\right]$} & Light yellow & 220 & 29.9 & $\begin{array}{c}11.97 \\
(11.63)\end{array}$ & 27 & Dia \\
\hline
\end{tabular}

*decomposition 
Table 2: IR bands and electronic spectral data of ligand and prepared complexes

\begin{tabular}{|c|c|c|c|c|c|}
\hline \multirow{2}{*}{ No. } & \multirow{2}{*}{ Compound } & \multirow{2}{*}{ U-Vis. bands $\left(\mathrm{cm}^{-1}\right)$} & \multicolumn{3}{|c|}{ IR spectra $\left(\mathrm{cm}^{-1}\right)$} \\
\hline & & & $v(C-O)$ & $v(C=S)$ & $\mathrm{v}(\mathrm{M}-\mathrm{S})$ \\
\hline & (2-BuoEtXant)K & --- & 1028 & 1099 & --- \\
\hline 1 & {$\left[\mathrm{Fe}(2-\mathrm{BuoEtXant})_{2}\right]$} & $9842,35714,38167$ & 1259 & 1039 & 437 \\
\hline 2 & {$\left[\mathrm{Fe}(2-\mathrm{BuoEtXant})_{2} \cdot(\mathrm{py})_{2}\right]$} & $11037,34482,35714$ & 1200 & 1039 & 435 \\
\hline 3 & {$\left[\mathrm{Fe}(2-\mathrm{BuoEtXant})_{2} .(4-\mathrm{pic})_{2}\right]$} & $11037,28248,34965,37593$ & 1188 & 1053 & 430 \\
\hline 4 & {$\left[\mathrm{Fe}(2-\mathrm{BuoEtXant})_{2} \cdot(\text { iso-Q })_{2}\right]$} & $10224,28409,32051$ & 1182 & 1059 & 434 \\
\hline 5 & {$\left[\mathrm{Fe}(2 \text {-BuoEtXant })_{2} \cdot(3,5 \text {-lut })_{2}\right]$} & $11037,35460,37313$ & 1244 & 1070 & 432 \\
\hline 6 & {$\left[\mathrm{Fe}(2-\mathrm{BuoEtXant})_{2} \cdot(1,10\right.$-phen $\left.)\right]$} & $10823,30120,31645$ & 1188 & 1065 & 422 \\
\hline 7 & {$\left[\mathrm{Fe}(2-\mathrm{BuoEtXant})_{2} \cdot(\mathrm{en})\right]$} & $10217,34482,35714$ & 1180 & 1043 & 434 \\
\hline 8 & {$\left[\mathrm{Co}(2 \text {-BuoEtXant })_{2}\right]$} & $15544,32051,35211$ & 1250 & 1031 & 430 \\
\hline 9 & {$\left[\mathrm{Co}(2 \text {-BuoEtXant })_{2} \cdot(\text { py })_{2}\right]$} & $10183,15708,21777,34722$ & 1221 & 1057 & 415 \\
\hline 10 & {$\left[\mathrm{Co}(2-\mathrm{BuoEtXant})_{2} .(4-\text { pic })_{2}\right]$} & $11037,15915,20455,31250,32051$ & 1219 & 1041 & 426 \\
\hline 11 & {$\left[\mathrm{Co}(2-\mathrm{BuoEtXant})_{2} \cdot(\text { iso-Q })_{2}\right]$} & $11086,15845,20595,31055,35211$ & 1217 & 1039 & 420 \\
\hline 12 & {$\left[\mathrm{Co}(2 \text {-BuoEtXant })_{2} \cdot(3,5 \text {-lut })_{2}\right]$} & $9823,15708,21472,32467,37593$ & 1219 & 1057 & 419 \\
\hline 13 & {$\left[\mathrm{Co}(2-\text {-BuoEtXant })_{2 .} \cdot(1,10\right.$-phen $\left.)\right]$} & $11061,16339,20024,34722$ & 1159 & 1057 & 421 \\
\hline 14 & {$\left[\mathrm{Co}(2-\mathrm{BuoEtXant})_{2}\right.$. (en) $]$} & $10224,14124,21624,30674$ & 1165 & 1059 & 420 \\
\hline 15 & {$\left[\mathrm{Ni}(2 \text {-BuoEtXant })_{2}\right]$} & $9433,15290,28571,30864$ & 1269 & 1051 & 440 \\
\hline 16 & {$\left[\mathrm{Ni}(2 \text {-BuoEtXant })_{2} \cdot(\mathrm{py})_{2}\right]$} & $9363,15197,24752,29761,32896$ & 1201 & 1057 & 438 \\
\hline 17 & {$\left[\mathrm{Ni}(2-\mathrm{BuoEtXant})_{2 \cdot} \cdot(4-\mathrm{pic})_{2}\right]$} & $9398,15723,26069,31847$ & 1194 & 1061 & 430 \\
\hline 18 & {$\left[\mathrm{Ni}(2-\mathrm{BuoEtXant})_{2} \cdot(\text { iso-Q })_{2}\right]$} & $9433,15243,25510,29940$ & 1186 & 1061 & 434 \\
\hline 19 & {$\left[\mathrm{Ni}(2 \text {-BuoEtXant })_{2} \cdot(3,5 \text {-lut })_{2}\right]$} & $9433,15290,26041,29585$ & 1203 & 1061 & 426 \\
\hline 20 & {$\left[\mathrm{Ni}(2 \text {-BuoEtXant })_{2} \cdot(1,10\right.$-phen $\left.)\right]$} & $10460,16447,27472,29411$ & 1188 & 1063 & 424 \\
\hline 21 & {$\left[\mathrm{Ni}(2-\mathrm{BuoEtXant})_{2 .} \cdot(\mathrm{en})\right]$} & $9609,16037,21739,30674,31847$ & 1157 & 1028 & 425 \\
\hline 22 & {$\left[\mathrm{Cu}(2-\mathrm{BuoEtXant})_{2}\right]$} & $9823,27777,34246$ & 1209 & 1038 & 432 \\
\hline 23 & {$\left[\mathrm{Cu}(2 \text {-BuoEtXant })_{2} \cdot(\text { py })_{2}\right]$} & $13157,29585,32467$ & 1132 & 1039 & 418 \\
\hline 24 & {$\left[\mathrm{Cu}(2-\text { BuoEtXant })_{2} \cdot(4-\text { pic })_{2}\right]$} & $13157,32258,33112$ & 1130 & 1038 & 420 \\
\hline 25 & $\left.\left[\mathrm{Cu}(2-\mathrm{BuoEtXant})_{2 .} \text { (iso-Q }\right)_{2}\right]$ & $13054,25000,31847$ & 1130 & 1038 & 422 \\
\hline 26 & {$\left[\mathrm{Cu}(2 \text {-BuoEtXant })_{2} \cdot(3,5 \text {-lut })_{2}\right]$} & $13477,29940,31847$ & 1132 & 1038 & 424 \\
\hline 27 & {$\left[\mathrm{Cu}(2 \text {-BuoEtXant })_{2} \cdot(1,10\right.$-phen $\left.)\right]$} & $13886,25641,32679$ & 1132 & 1038 & 430 \\
\hline 28 & {$\left[\mathrm{Cu}(2-\mathrm{BuoEtXant})_{2} .(\mathrm{en})\right]$} & $13376,24154,34965$ & 1188 & 1050 & 418 \\
\hline 29 & {$\left[\mathrm{Zn}(2 \text {-BuoEtXant })_{2}\right]$} & --- & 1215 & 1061 & 430 \\
\hline 30 & {$\left[\mathrm{Zn}(2 \text {-BuoEtXant })_{2} \cdot(\mathrm{py})_{2}\right]$} & --- & 1201 & 1068 & 418 \\
\hline 31 & {$\left[\mathrm{Zn}(2 \text {-BuoEtXant })_{2} \cdot(4 \text {-pic })_{2}\right]$} & --- & 1173 & 1068 & 419 \\
\hline 32 & $\left.\left[\mathrm{Zn}(2-\mathrm{BuoEtXant})_{2 .} \text { (iso-Q }\right)_{2}\right]$ & --- & 1201 & 1059 & 421 \\
\hline 33 & {$\left[\mathrm{Zn}(2 \text {-BuoEtXant })_{2} \cdot(3,5-\operatorname{lut})_{2}\right]$} & -- & 1173 & 1068 & 420 \\
\hline 34 & {$\left[\mathrm{Zn}(2 \text {-BuoEtXant })_{2} \cdot(1,10\right.$-phen $\left.)\right]$} & --- & 1144 & 1070 & 418 \\
\hline 35 & {$\left[\mathrm{Zn}(2 \text {-BuoEtXant })_{2}\right.$.(en)] } & $\begin{array}{c}-- \\
\end{array}$ & 1144 & 1072 & 422 \\
\hline
\end{tabular}




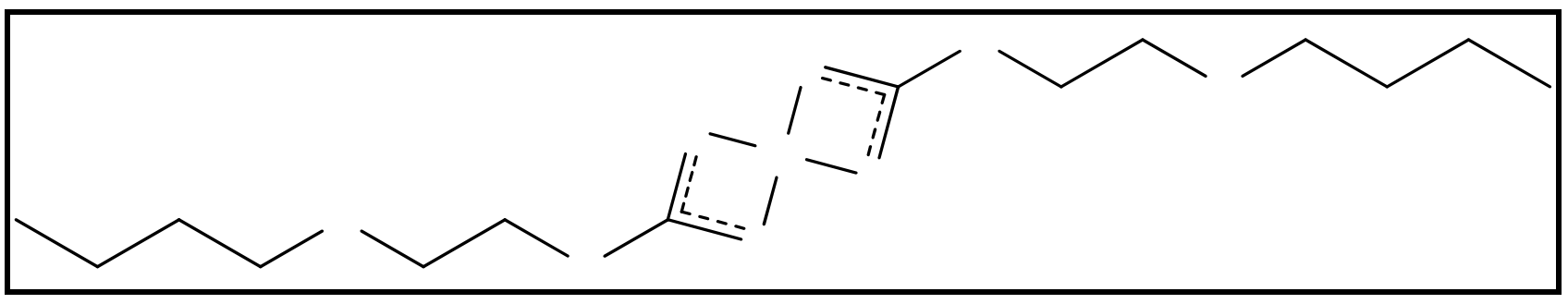

(a)

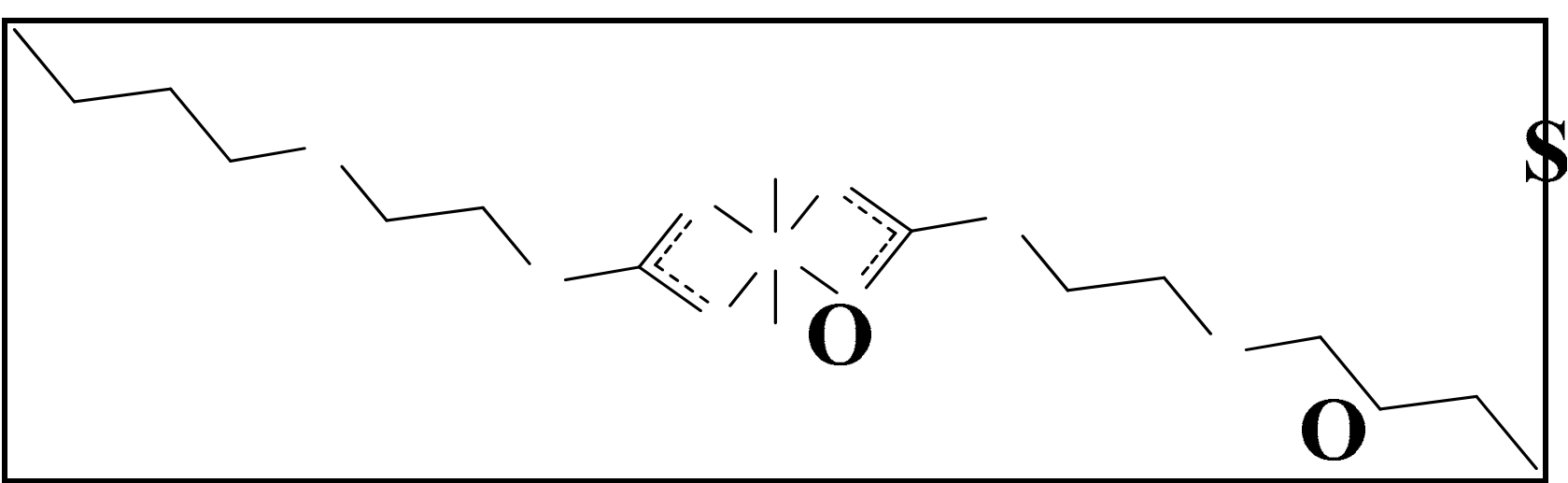

(b)
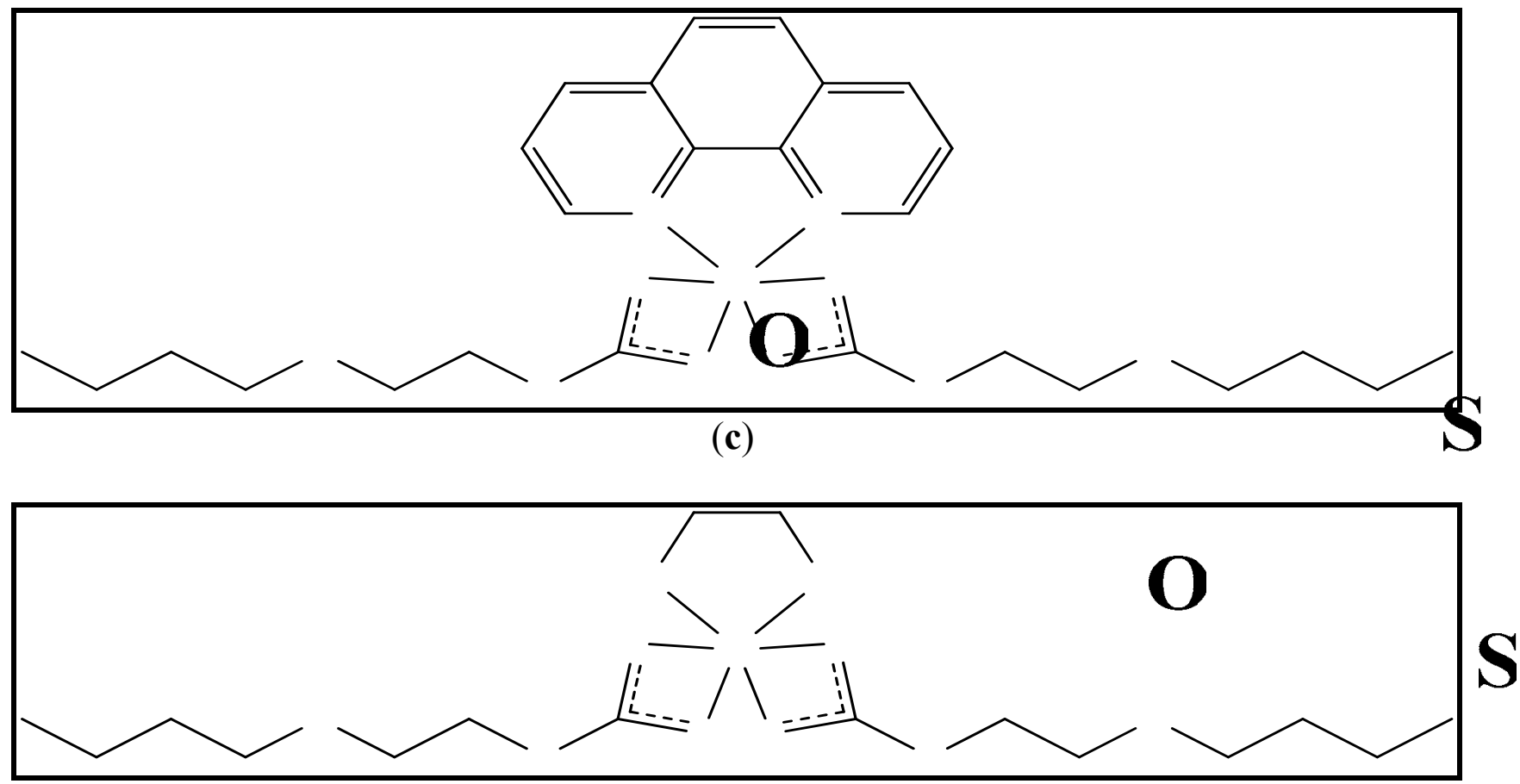

$\mathbf{N}$

$\mathbf{S}$

(d)

Fig. 1: Suggest structures (a) complexes $[1,8,15,22,29]$, (b) complexes $[2-5,9-12,16-$ 19, 23-26, 30-33], (c) complexes $[6,13,20,27,34]$, (d) complexes[7, 14, 21, 28, 35]

\section{REFERENCES}

Bond, A.M.; Martin, R.L. (1984). Electrochemistry and redox behaviour of transition metal dithiolate. Coord. Chem. Rev., 54, 23. 
Cookson, J.; Emma, A.L.E.; John, P.M.; Christopher, J.S.; Rowena, L.P.; Andrew, R.C.; Michael, G.B.D.; Paul, D. (2010). Metal directed assembly of large dinuclear copper(II) dithiocarbamate macrocyclic complexes. Inorg Chem. Acta., 363, 1195-1203.

Coote, M.L.; Radom, L. (2004). Substituent effects in xanthate-mediated polymerization of vinyl acetate: abinitio evidence for an alternative fragmentation pathway. Macromolecules., 37, 590-596.

Coucouvanis, D.; Fackler, J.P. (1967). Square-planar sulfur complexes.VI. reactions of bases with xanthate, dithiocarbamates, and dithiolates of nickel(II). J. Inorg. Chem., 6, 20472053.

Coucouvanis, D. (1970). Coordination chemistry of dithio ligands, Prog. Inorg. Chem., 11, 233,179 .

Coucouvanis, D. (1979). The chemistry of dithio acid and 1,1-dithiolate complexes. Prog. Inorg. Chem., 26, 301, 274.

Cox, M.J.; Tiekink, E.R. (1997). The diverse coordination patterns in the structures of zinc, cadmium and mercury bis (1,1-dithiolates). Rev. Inorg. Chem., 17, 1.

Geary, W.J. (1971). The use of conductivity measurements in organic solvents for characterization of coordination compounds. Coord. Chem. Rev.7, 8-122.

Hill, J.O. (1994). 30 years of research in thermal analysis and calorimetry. J. Therm. Analy., 42, 607-621.

Lever, A.B.P. (1984). "Inorganic Electronic Spectroscopy". 2nd ed., Elsevier, Amsterdam, $357 \mathrm{p}$.

Magnus, K.A.; Ton-That, H.; Carpenter, J.E. (1994). Recent Structure work on the oxygen transport proliein Hemo cyanin. Chem. Rev., 94, 727-735.

Martell, A.E. (1971). "Coordination Chemistry". Van Nostrand Reinhold, New York., 1 p.

Nicholls, D. (1973). "The Chemistry of Iron, Cobalt and Nickel". 1st ed., Pergamon press, Oxford, pp.1037, 1087, 1088,1090, 1091,1093, 1151,1154.

Onwudiwe, D. C.; Ajibade, P.A.(2010). Synthesis and characterization of metal complexes of N-alkyl-N-phenyl dithiocarbamates. Polyhedron .,29,1431-1436.

Rathore, H.S.; Varshney, G.; Mojumdar, S.C.; Saleh, M.T. (2007). Synthesis, charcterization and fungicidal activity of zinc diethyldithIocarbamate and phosphate. Ther. Anal. and Calorim, 90(3), 681-686..

Sarwar, M.; Ahmad, S.; Ahmad, S.; Ali, S.; Awan, S.A. (2007). Copper(II) complexes of pyrrolidine dithiocarbamate. Trans Met Chem., 32,199-203.

Serrano, J.L.; Garcı'a, L.; Pe'rez, J.; Pe'rez, E.; Sa'nchez, G.; Garcı'a, J.; Lo'pez, G.; Garcı'a, G.; Molins, E. (2003). New dithiocarbamate and xanthate complexes of nickel(II) with iminophosphines. Inorg. Chem. Acta ., 355, 33- 40.

Siddiqi, K.S.; Nishat, N. (2000). Synthesis and characterization of succinimide and phthalimide dithiocarbamate and their complexes with some transition metal ions. Synth. Reac. Inorg. Met-Org. Chem., 30(8), 1505 -1518.

Singh, N.; Singh, N.K.; Kaw, C. (1989). Synthetic and spectroscopic studies of xanthatebridged heterobimetallic complexes containing diamagnetic and paramagnetic metal ions. Bull. Chem. Soc. (Jpn.), 62, 3328-3333.

Tiekink, E.R.T.; Haiduc, I. (2005). Stereochemical aspects of metal xanthate complexes molecular stractures and supramolecular selfassembly. Prog. Inorg. Chem., 54,127-319.

Wan, D.C.; Sato, K.; Kamigaito, M.; Okamoto, Y. (2005). Xanthate- mediated radical polymerization of $\mathrm{N}$-vinyl pyrrolidone in fluoroacoholes for simultaneous control of Molecular weight and tacicity. Macromolecules., 38,10397-10405. 